Journal of Soft Computing Paradigm (JSCP) (2019)

Vol.01/ No. 02

Pages: $57-68$

http://irojournals.com/jscp/

DOI: https://doi.org/10.36548/jscp.2019.2.001

\title{
PERFORMANCE ENHANCEMENTS OF COGNITIVE RADIO NETWORKS USING THE IMPROVED FUZZY LOGIC
}

\author{
Dr. P Ebby Darney \\ Professor, Department of EEE, \\ SCAD CET. \\ darney.pebby@gmail.com \\ Dr. I. Jeena Jacob, \\ Department of Computer Science and Engineering, \\ GITAM University, Bangalore, India. \\ jeenajacob2016@gmail.com
}

\begin{abstract}
The rapid increase in the mobile device and the different types of wireless communication has led to the necessity of the extra spectrum allocation for the proper transmission of the information. Since the additional spectrum allocation for every network involved in the data transmission is a strenuous process, the efficient management of the spectrum allocation is preferred. The cognitive radio technology does a befitting service in the managing the allocation of the spectrum efficiently by providing the vacant spaces of the licensed users to the secondary users and vacating the secondary users when the licensed user request for the spectrum. This results in the deterioration in the performance of the secondary users due to the immediate evacuating. The conventional methods in the deciding the channel switching remains unsuitable for the cognitive radio network, so to have an effective decision on switching and selecting the channel the paper put forth the improved fuzzy logic that relies on the decision (IFDSS-GA) support system to handle both the switching of the channels and genetic algorithm to select the proper spectrum for conveyance. The evaluation of the proposed approach using the network simulator -2 determines the competency the IFDSS in terms of the throughput and switching rate.
\end{abstract}

Keywords: Improved Fuzzy Logic, Cognitive Radio Technology, Decision Support Systems, Channel Switching, Throughput and Switching Rate

\section{INTRODUCTION}

The tremendous improvement and the advancement in the wireless technology have paved way novel researches that provide a captivating out comes. But the results obtained are never a solution as the progressing growth in the technologies lead to more and more issues and challenges to the researcher's. One such case is the spectrum allocation for the mobile devices; the mobile device and the users keep on increasing day by day and the rate of conveyance increases exponentially. To have an effective spectrum allocation the cognitive radio networks are employed. The cognitive radio network identifies the unused space in the bands of the licensed users and enables it

ISSN: 2582-2640 (online)

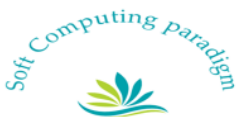


Journal of Soft Computing Paradigm (JSCP) (2019)

Vol.01/ No. 02

Pages: $57-68$

http://irojournals.com/jscp/

DOI: https://doi.org/10.36548/jscp.2019.2.001

to be used by the secondary users and later reallocates the secondary users to the free space in the next band whenever a transmission is initiated by licensed user.

The cognitive radio networks are basically categorized into two types as shown below.

(i). Centralized-Cognitive radio network

(ii) Decentralized-Cognitive radio network

\section{(i). CENTRALIZED-CRN}

It is an infrastructure-based network with its secondary users being managed by separately employing a base station specifically for them. The connections in them are managed by a wired back bone. The fig. 1 below shows the frame work of the centralized $-\mathrm{CRN}$.

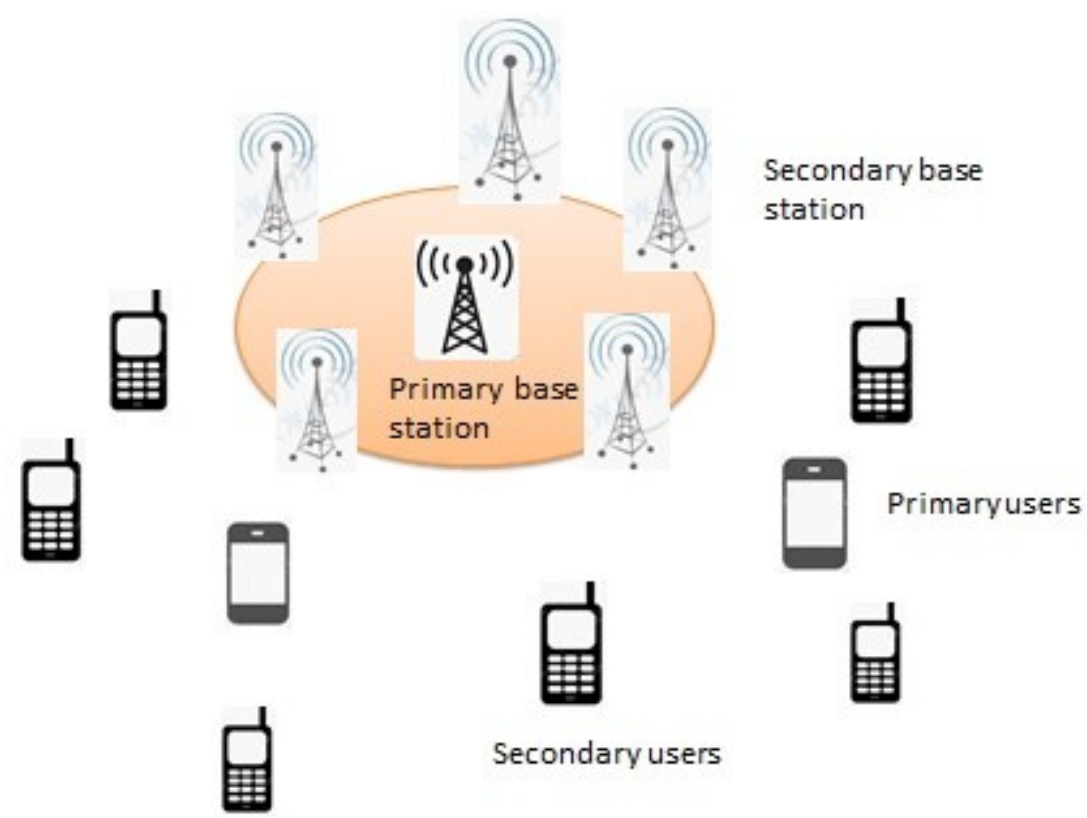

Fig.1 Centralized-CRN

ISSN: 2582-2640 (online)

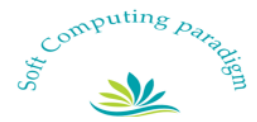


Journal of Soft Computing Paradigm (JSCP) (2019)

Vol.01/ No. 02

Pages: $57-68$

http://irojournals.com/jscp/

DOI: https://doi.org/10.36548/jscp.2019.2.001

\section{(ii). Decentralized-CRN}

The Decentralized- CRN are frame in adhoc manner, where the secondary users extend communication in an adhoc way. Its spectrum scanning operation is generally done in a cooperative way. This framework enables more than one user to be operating under the band that is unlicensed. A well-known example is the simultaneous existence of the IEEE802.11 with the IEEE 802.16. The fig.2 below provides the frame work of the decentralized-CRN.

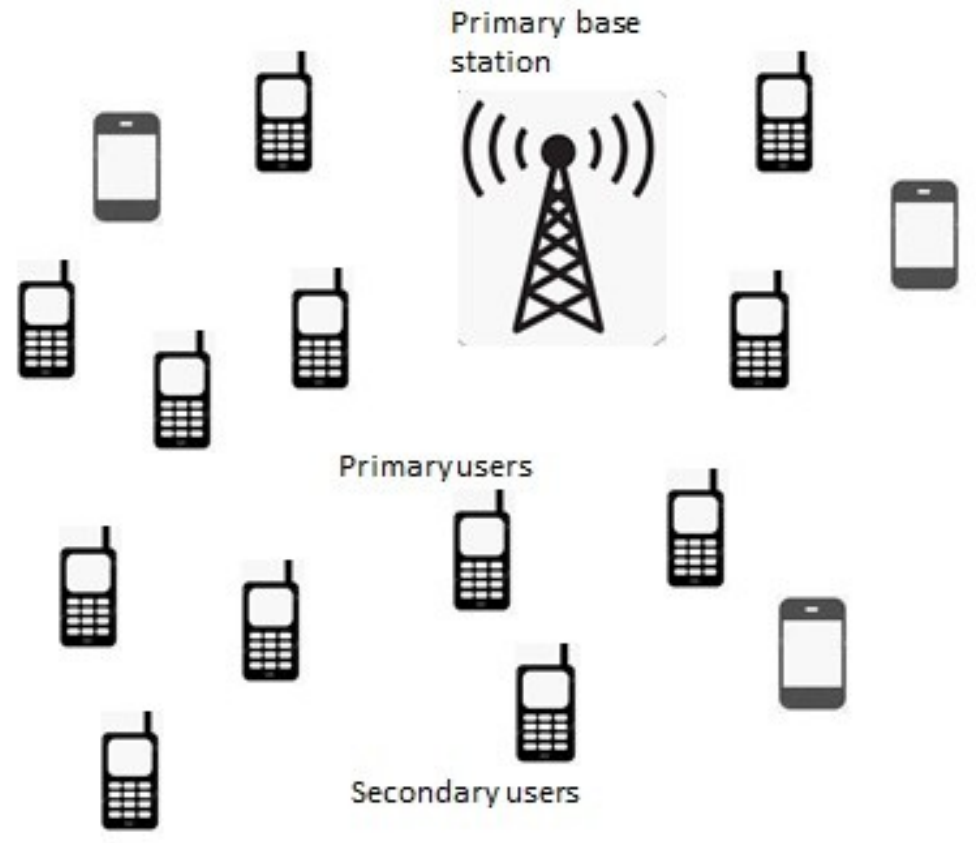

Fig.2 Decentralized-CRN

The cognitive radio networks resulted in poor performance due to the challenges in the spectrum sensing, end to end quality services and mobility. The employments of the conventional methods were unsuitable for providing perfect selection and switching bands for the secondary users.

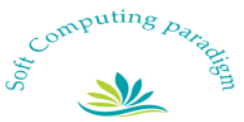


Journal of Soft Computing Paradigm (JSCP) (2019)

Vol.01/ No. 02

Pages: $57-68$

http://irojournals.com/jscp/

DOI: https://doi.org/10.36548/jscp.2019.2.001

So the paper put forth the IFDSS-GA utilizing the genetic algorithm to identify the appropriate channel with the minimum signal to noise ratio, link error rate, delays, holding time and the interference and utilizes the IFDSS decision support integrated with the fuzzy to decide the switching.

The proposed method mainly concentrates on reducing the number of switching for the secondary users and enhancing the throughput of the cognitive radio networks by making the channel switching more adaptable.

The paper below is arranged with the related works explaining the employment of the fuzzy logic in the cognitive radio network in 2, the proposed work employing the IFDSS-GA for channel selection and switching in cognitive radio network in 3 , the result analysis in 4 , and conclusion in 5 .

\section{RELATED WORKS}

Baldo et al [1], author put forth the fuzzy logic design to as a befitting way for identifying an $\mathrm{d}$ as well as solving the challenges such as the " modularity, imprecision, interpretability, scalability, and complexity constraints in search of the in optimal frame work for the "cross layer optimization in the CRN" ."A fuzzy decision scheme for cooperative spectrum sensing in cognitive radio." Proffered by the author Zhang, et al [2], presents the utilization of the "closed form expression between the probabilities of detecting and the false alarm for the centralized cooperative network in the cognitive radio network that incorporates the fuzzy decision scheme"

"Cognitive network access using fuzzy decision making." Put forth by the Baldo,et al [3] explains the usage of the "distributed cognitive radio network in providing a best quality of service for the internet users who wish to connect utilizing the several available network access and compares the estimated to the requirements of the application using the fuzzy decision making.

Niyato et al [4] elaborates the various components and the related approaches available to gain the adaptability in the cognitive radio networks. And presents the "dynamic opportunistic channel scheme based on the cognitive radio concept for the IEEE802.11 WMN."

Mathad et al [5] the paper presents the "over view of the cross layer design and introduces the approaches utilized in the literatures along with the methods of the cross-layering based on the classical optimization techniques, the survey provides the details of the various schemes for improving of the performance in the CRN'

ISSN: 2582-2640 (online)

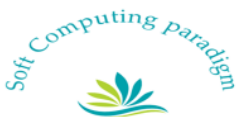


Journal of Soft Computing Paradigm (JSCP) (2019)

Vol.01/ No. 02

Pages: $57-68$

http://irojournals.com/jscp/

DOI: https://doi.org/10.36548/jscp.2019.2.001

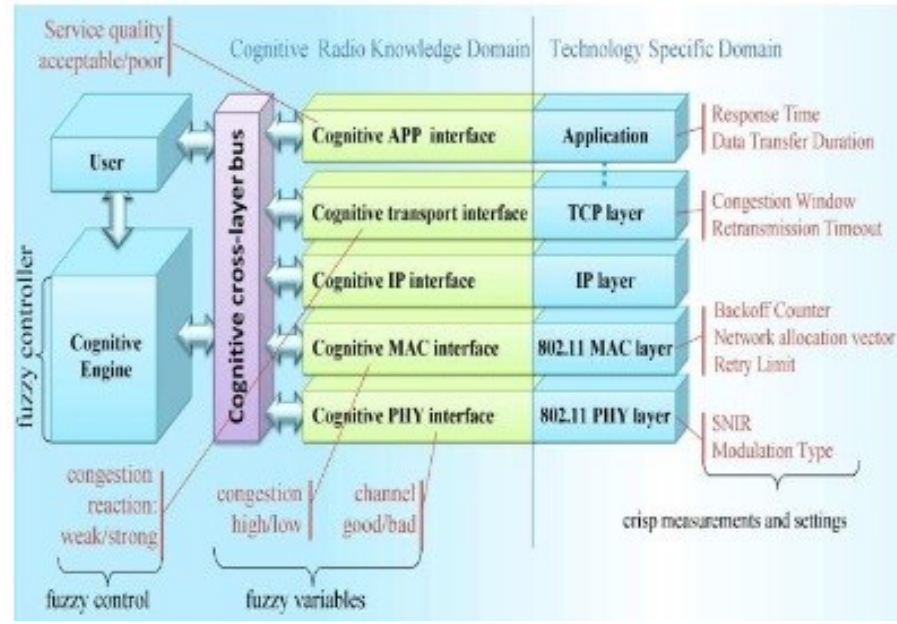

Fig..3 Fuzzy logic in Cross Layering [5]

Joshi et al [6] the survey provides the information related to the emerging cognitive radio sensor network and its applications highlights its advantages, the paper provides the comparison between the, CR-WSN , Adhoc- CRN, WSN along with the area where they are put in use

Ejaz,et al [7] the author utilizes the fuzzy logic in identifying the unused portion in the cognitive radio network, employing the conventional methods in the initial stage followed by the fuzzy logic to decide the unused portion that is the absence of the licensed users.

El Masri et al [8] the author utilizes the fuzzy logic along with the stability metric and the predicted power metric to have an appropriate routing decision in the cognitive radio networks Matinmikko et al [9] explores the "Fuzzy-logic based framework for spectrum availability assessment in cognitive radio systems" For having an improved way of communication.

PARK et al [10] puts forth the "FOREWORDApplication of Fuzzy Logic to Cognitive Radio SystemsDynamic Spectrum Access to the Combined Resource of Commercial and Public Safety Bands Based on a WCDMA Shared NetworkDynamic Resource Allocation in OFDMA Systems with Adjustable QoSA Novel Dynamic Channel Access Scheme Using Overlap FFT Filter-Bank for Cognitive RadioPerformance Analysis of Control Signal Transmission Technique for Cognitive Radios in Dynamic Spectrum Access NetworksSpectrum Sensing Architecture and Use Case Study"

ISSN: 2582-2640 (online)

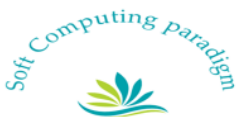


Journal of Soft Computing Paradigm (JSCP) (2019)

Vol.01/ No. 02

Pages: $57-68$

http://irojournals.com/jscp/

DOI: https://doi.org/10.36548/jscp.2019.2.001

The "Analysis and Comparison of Different Fuzzy Inference Systems Used in Decision Making for Secondary Users in Cognitive Radio Network" is presented by Tripathi, et al [11] and Banerjee et al [12] provides the "Joint cooperative spectrum sensing and primary user emulation attack detection in cognitive radio networks using fuzzy conditional entropy maximization."

The author Alhammadi, et al [13] puts forwards "An intelligent spectrum handoff scheme based on multiple attribute decision making for LTE-A network." While the Ekti, et al [14] elaborates the utilization of the "Fuzzy Logic Approach for Layered Architecture Cognitive Radio Systems." Mathur et al [15], details the "Security Issues in Cognitive Radio Networks."

Thakur, et al [16] provides the "Performance analysis of high-traffic cognitive radio communication system using hybrid spectrum access, prediction and monitoring techniques" Ali et al [17] "Channel clustering and QoS level identification scheme for multi-channel cognitive radio networks." Liang et al [18] presents the "Cooperative overlay spectrum access in cognitive radio networks." Zhao et al [19] provides the "the spectrum allocation in the cognitive radio using the evolutionary algorithms."

\section{PROPOSED WORK}

The primary entailment in a cognitive radio network is its capability to identify the free spectrum to handle the switching of the users and improve the efficiency of the spectrum utilization. The proposed IFDSS-GA aims at having a more adjusted switching, by reducing the switching rate of the secondary users utilizing the genetic algorithm in selection of the spectrum and engaging the fuzzy logic in hand -off, the proffered method combines the interference avoiding approach (IAA) put forward by Thakur et al 2015 and the overlay method of the Ali et al 2018 to enable the secondary users to convey simultaneously along with the licensed users, during the absence as well as presence of the primary users. The fig. 4 below shows the general and the proffered spectrum allocation process of the cognitive radio network.

ISSN: 2582-2640 (online)

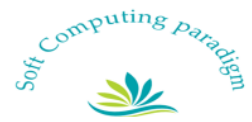


Journal of Soft Computing Paradigm (JSCP) (2019)

Vol.01/ No. 02

Pages: $57-68$

http://irojournals.com/jscp/

DOI: https://doi.org/10.36548/jscp.2019.2.001

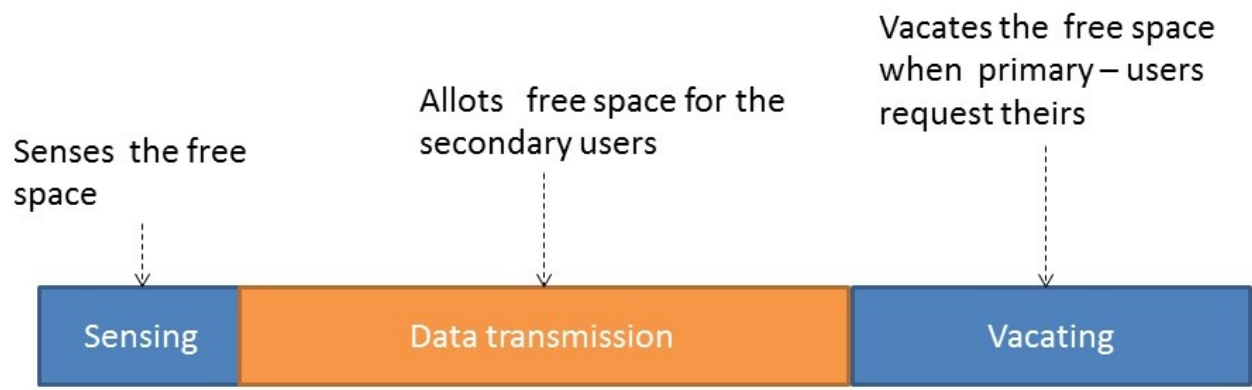

Fig. 4 (a) General Frame work of CRN

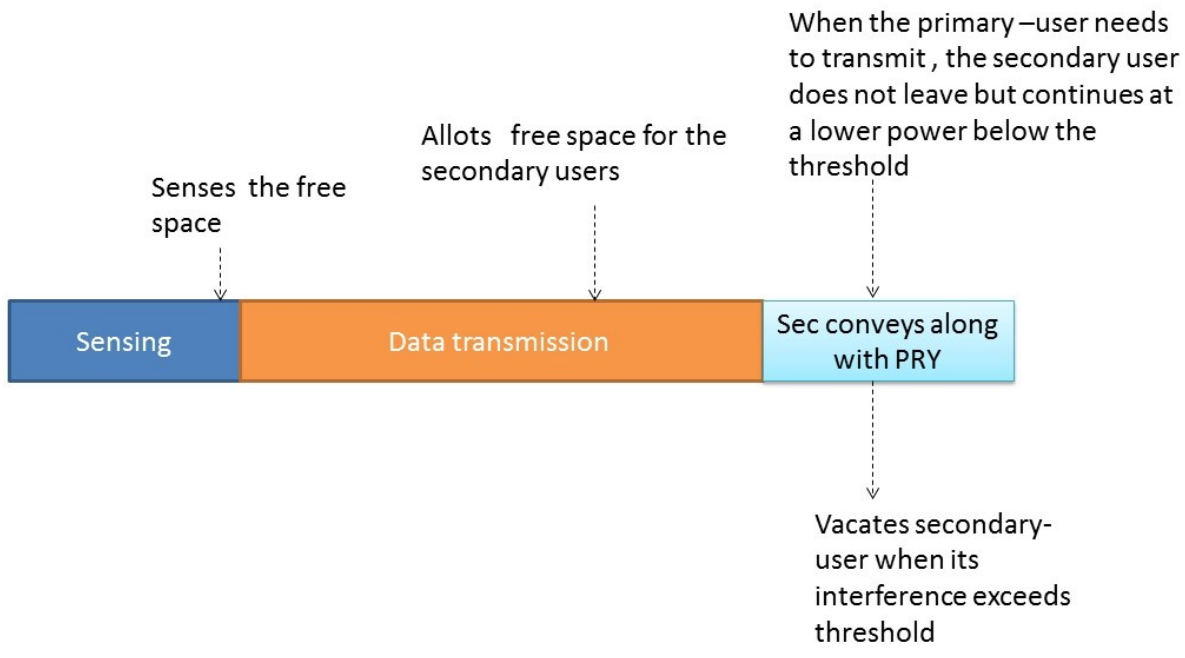

Fig 4(b) Proffered frame work of CRN combining the IAA and Overlay.

The integration of the IAA and the overlay enables the secondary-user to convey its information in the absence of the primary-users and the overlay enables the secondary user continue transmission in the presence of the primaryconsumer at a lower conveyance power that is within the onset level that is set.

The proposed model uses a decentralized-CRN in which the secondary-consumers are arranged and as well as communicate in an adhoc manner. The model in the proffered system is comprised of ' $i$ ' pry-consumers and the ' $j$ '

ISSN: 2582-2640 (online)

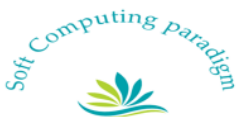


Journal of Soft Computing Paradigm (JSCP) (2019)

Vol.01/ No. 02

Pages: $57-68$

http://irojournals.com/jscp/

DOI: https://doi.org/10.36548/jscp.2019.2.001

sec-consumers. The sec-consumers hold a list of channels that can be used and updates it regularly, the time interval between the periodic updation is denoted as' $T$ '.

\subsection{CHANNEL SELECTION USING GENETIC ALGORITHM}

The genetic algorithm [19] is utilized in the proposed process to select the appropriate channel suitable for the communication evaluating and identifying the channels with the minimum signal to noise ratio, link error rate, delays, holding time and the interference. The evolutionary algorithm based on the population is used to evaluate the channels available, identify the befitting channels and updates it to the list. The steps below show the application of the genetic algorithm in the selection of the channel.

Step 1: Initialize the spectrums available; gather the information of the spectrum signal to noise ratio (SNR), link error rate $(L E R)$, delays $(D)$, holding time $(H T)$ and the interference $(I)$.

Step 2: Evaluate the fitness of the channel enumerating the levels of the $S N R, L E R, D, H T, I$ if optimal solution obtained, the convergence occurs and updates the optimal channel else.

Step 3: Apply cross over (1) and mutation (.5), and evaluate fitness, if optimal solution found, update the solution to the list.

Step 4: Check If maximum iterations leading to global convergence achieved stop else GOTO step 2.

The process continues until the all the optimal channels are identified and updated to the list.

\subsection{FUZZY INTERFERENCE FOR A PERFECT HAND-OFF}

The fuzzy interference system [11] is utilized in the proposed process to identify the interference level of the secondary users. The proposed method utilizes the combination of the IAA and the overlay to enable the secondaryconsumers to continue transmission in the presence and the absence of the licensed users. In the absence of the licensed user the secondary-consumers uses the channel for conveying and in the presence of the licensed-consumer the channel is shared with the secondary-consumer only if its inference level is below the threshold. In order to note down the interference level of the secondary users the paper utilizes the fuzzy interference system. On detecting the interference level beyond the threshold level, the fuzzy interference system completely evacuates the secondaryconsumers and does a perfect hand-off to the primary-consumers as shown in the equation (1). The interference level fed to the fuzzy is system is fuzzified using the Mamdani fuzzy interference system applying the triangular

ISSN: 2582-2640 (online)

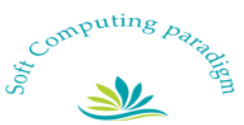


Journal of Soft Computing Paradigm (JSCP) (2019)

Vol.01/ No. 02

Pages: $57-68$

http://irojournals.com/jscp/

DOI: https://doi.org/10.36548/jscp.2019.2.001

membership function. The based threshold level that is fed to the knowledge base, the de-fuzzified values are obtained at the output. This enables the switching to be adaptive and done only when the interference level of the secondary -consumer is beyond the verge level. So this reduces the frequent switching of the secondary- users and enhances the performance of the $\mathrm{CRN}$ in terms of throughput.

$$
\text { hand }_{\text {off }} \text { if }\left\{\text { interfernce level }_{\text {sec-user }} \gg \text { Treshold }_{\text {level }}\right.
$$

\section{RESULTS ANALYSIS}

The evaluation of the IFDSS-GA is done using the network simulator -2 to note down the performance enhancement in the CRN in terms throughput and the switching rate. The results obtained are shown below in the fig. 5 . The result evinces the enhanced quality of service in the CRN by the application of IFDSS-GA for deciding the selection and the switching process for various numbers of secondary-users.

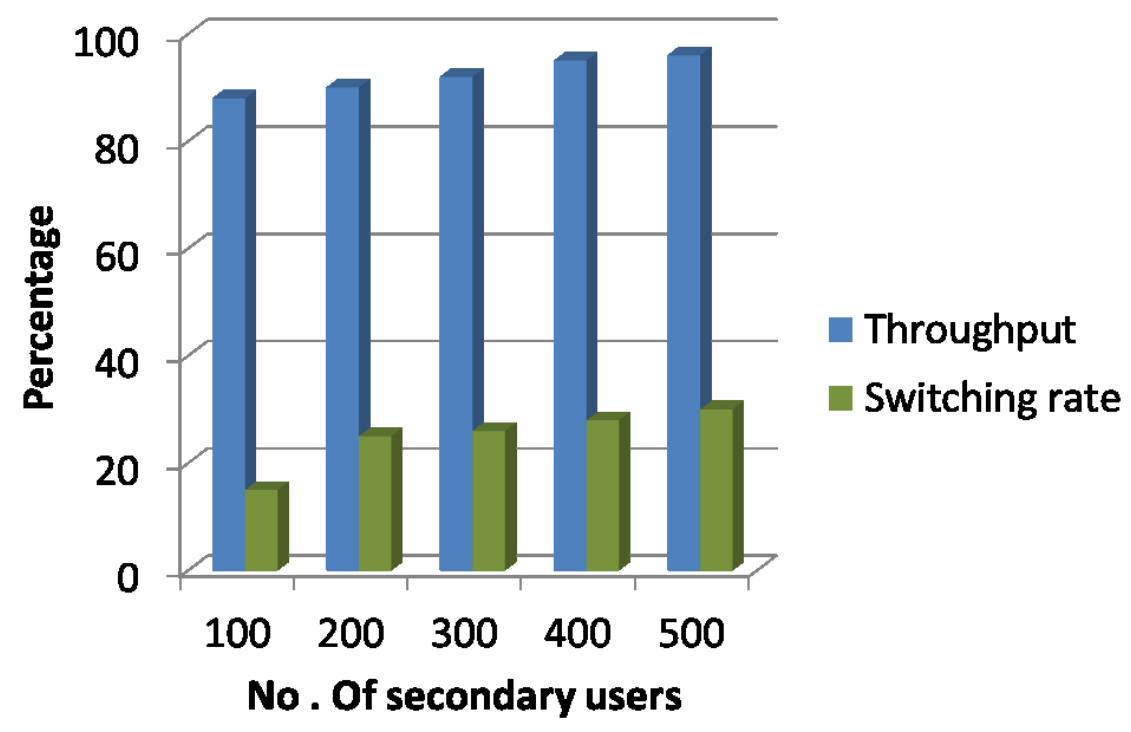

Fig.5 Performance Enhancement

The table.1 below shows the comparison of the IFDSS-GA with the other methods conventional methods utilized for the selecting and switching of the channel for different set of secondary users.

ISSN: 2582-2640 (online)

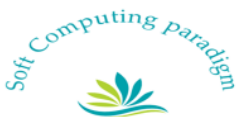


Journal of Soft Computing Paradigm (JSCP) (2019)

Vol.01/ No. 02

Pages: $57-68$

http://irojournals.com/jscp/

DOI: https://doi.org/10.36548/jscp.2019.2.001

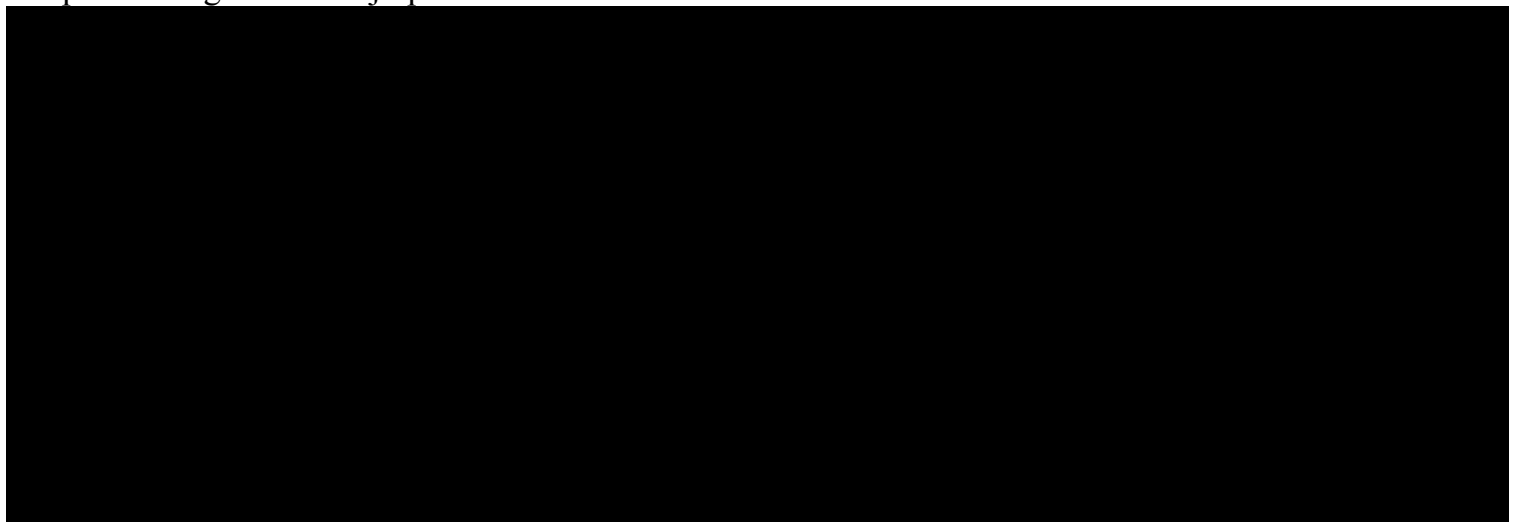

Table .1 Comparison of IFDSS-GA with Conventional

\section{CONCLUSION}

The paper employs the fuzzy interference system and the genetic algorithm to have a perfect decision on switching and selecting respectively of the spectrum in cognitive radio network for the mobile users who require additional channel access. The genetic algorithm is used to select the appropriate befitting channel that is suitable for communication by enumerating the channel with the minimum signal to noise ratio, link error rate, delays, holding time and the interference. Further the fuzzy interference is applied to the enumerate the interference level of the secondary user to decide whether the hand-off is necessitated or not. The combination of the IAA and the overlay in channel allocation along with the fuzzy interference system brings down the switching rate of the secondaryusers/consumers thus enhancing the overall performance of the secondary-users as well the CRN. The results obtained and the comparison proves the effective ness of the proposed method compared to the conventional method.

\section{References}

[1] Baldo, Nicola, and Michele Zorzi. "Fuzzy logic for cross-layer optimization in cognitive radio networks." IEEE Communications magazine 46, no. 4 (2008): 64-71.

[2] Zhang, Hongtao, and Xiaoxiang Wang. "A fuzzy decision scheme for cooperative spectrum sensing in cognitive radio." In 2011 IEEE 73rd Vehicular Technology Conference (VTC Spring), pp. 1-4. IEEE, 2011.

ISSN: 2582-2640 (online)

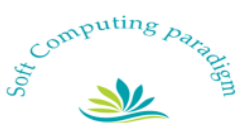


Journal of Soft Computing Paradigm (JSCP) (2019)

Vol.01/ No. 02

Pages: $57-68$

http://irojournals.com/jscp/

DOI: https://doi.org/10.36548/jscp.2019.2.001

[3] Baldo, Nicola, and Michele Zorzi. "Cognitive network access using fuzzy decision making." IEEE Transactions on Wireless Communications 8, no. 7 (2009): 3523-3535.

[4] Niyato, Dusit, and Ekram Hossain. "Cognitive radio for next-generation wireless networks: An approach to opportunistic channel selection in IEEE 802.11-based wireless mesh." IEEE Wireless Communications 16, no. 1 (2009): 46-54.

[5] Mathad, Anandteerth, and Mrinal Sarvagya. "Cross layer design approaches, schemes and optimization methodologies for cognitive radio networks: a survey." Quest journals, Journal of electronics and communication engineering research 1, no. 4 (2013): 15-21.

[6] Joshi, Gyanendra Prasad, Seung Yeob Nam, and Sung Won Kim. "Cognitive radio wireless sensor networks: applications, challenges and research trends." Sensors 13, no. 9 (2013): 11196-11228.

[7] Ejaz, Waleed, Najam ul Hasan, Saleem Aslam, and Hyung Seok Kim. "Fuzzy logic based spectrum sensing for cognitive radio networks." In 2011 Fifth International Conference on Next Generation Mobile Applications, Services and Technologies, pp. 185-189. IEEE, 2011.

[8] El Masri, Ali, Naceur Malouch, and Hicham Khalife. "A routing strategy for cognitive radio networks using fuzzy logic decisions." In the proceedings of the first Conference Cognitive Advances in Cognitive Radio IARIA COCORA, pp. 1-14. 2011.

[9] Matinmikko, Marja, Javier Del Ser, Tapio Rauma, and Miia Mustonen. "Fuzzy-logic based framework for spectrum availability assessment in cognitive radio systems." IEEE Journal on Selected Areas in Communications 31, no. 11 (2013): 2173-2184.

[10] PARK, Chee-Hyun, and Kwang-Seok HONG. "FOREWORDApplication of Fuzzy Logic to Cognitive Radio SystemsDynamic Spectrum Access to the Combined Resource of Commercial and Public Safety Bands Based on a WCDMA Shared NetworkDynamic Resource Allocation in OFDMA Systems with Adjustable QoSA Novel Dynamic Channel Access Scheme Using Overlap FFT Filter-Bank for Cognitive RadioPerformance Analysis of Control Signal Transmission Technique for Cognitive Radios in Dynamic Spectrum Access NetworksSpectrum Sensing Architecture and Use Case Study ...."

[11] Tripathi, Shrivishal, Ashish Upadhyay, Shashank Kotyan, and Sandeep Yadav. "Analysis and Comparison of Different Fuzzy Inference Systems Used in Decision Making for Secondary Users in Cognitive Radio Network." Wireless Personal Communications 104, no. 3 (2019): 1175-1208.

[12] Banerjee, Avik, and Santi P. Maity. "Joint cooperative spectrum sensing and primary user emulation attack detection in cognitive radio networks using fuzzy conditional entropy maximization." Transactions on Emerging Telecommunications Technologies 30, no. 5 (2019): e3567.

[13] Alhammadi, Abdulraqeb, Mardeni Roslee, Mohamad Yusoff Alias, Khalid Sheikhidris, Yong Jun Jack, Anas Bin Abas, and Kesh S. Randhava. "An intelligent spectrum handoff scheme based on multiple attribute decision making for LTE-A network." International Journal of Electrical \& Computer Engineering (2088-8708) 9 (2019).

ISSN: 2582-2640 (online)

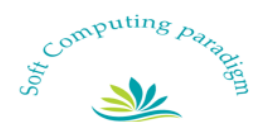


Journal of Soft Computing Paradigm (JSCP) (2019)

Vol.01/ No. 02

Pages: $57-68$

http://irojournals.com/jscp/

DOI: https://doi.org/10.36548/jscp.2019.2.001

[14] Ekti, Ali Riza. "Fuzzy Logic Approach for Layered Architecture Cognitive Radio Systems." In International Telecommunications Conference, pp. 61-71. Springer, Singapore, 2019.

[15] Mathur, Chetan N., and K. P. Subbalakshmi. "Security Issues in Cognitive Radio Networks." Cognitive Networks: Towards Self-Aware Networks (2007): 271.

[16] Thakur, Prabhat, Alok Kumar, Shweta Pandit, Ghanshyam Singh, and S. N. Satashia. "Performance analysis of high-traffic cognitive radio communication system using hybrid spectrum access, prediction and monitoring techniques." Wireless Networks 24, no. 6 (2018): 2005-2015.

[17] Ali, Amjad, Ibrar Yaqoob, Ejaz Ahmed, Muhammad Imran, Kyung Sup Kwak, Adnan Ahmad, Syed Asad Hussain, and Zulfiqar Ali. "Channel clustering and QoS level identification scheme for multichannel cognitive radio networks." IEEE Communications Magazine 56, no. 4 (2018): 164-171.

[18] Liang, Wei, Soon Xin Ng, and Lajos Hanzo. "Cooperative overlay spectrum access in cognitive radio networks." IEEE Communications Surveys \& Tutorials 19, no. 3 (2017): 1924-1944.

[19] Zhao, Zhijin, Zhen Peng, Shilian Zheng, and Junna Shang. "Cognitive radio spectrum allocation using evolutionary algorithms." IEEE Transactions on Wireless Communications 8, no. 9 (2009): 44214425.

ISSN: 2582-2640 (online)

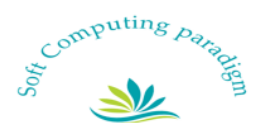

